\title{
DNA restriction fragment length polymorphism of HLA-DR2 haplotypes in normal individuals and in patients with rheumatoid arthritis
}

\author{
Dharam P Singal, Barbara Reid, Donna Green, William G Bensen, Merl D'Souza
}

\begin{abstract}
A strong association between HLA-DR4 and rheumatoid arthritis (RA) has been found in a number of populations. In contrast, the incidence of $D R 2$ is decreased in patients with RA, suggesting that this specificity may confer some protection against the disease. A number of subtypes of DR2 have been defined by serology, by responses in mixed lymphocyte culture reaction, and, more recently, by restriction fragment length polymorphism. These subtypes of DR2 are in linkage disequilibrium with different subspecificities of DQw1. It is thus likely that the distribution of these subtypic DR,DQ haplotypes in DR2 positive patients with RA may be important in understanding the genetic basis of susceptibility/resistance to RA. In this paper a study of the subtypes of DR2,DQw1 haplotypes in 18 patients with RA, who required sodium aurothiomalate as a disease remitting drug, and unrelated healthy individuals is reported. Three subtypes of DR2 haplotypes, DRw15 (Dw2),DQw1.2(DQw6), DRw15(Dw12),DQw1. 12(DQw6), and DRw16(Dw21),DQw1, AZH (DQw5), were analysed with a CDNA probe for the DQ $\beta$ gene. The data show that DR2 positive patients with RA carried either the DRw15(Dw2),DQw6 or DRw15(Dw12),DQw6 haplotype. No patient with RA was positive for the DRw16(Dw21),DQw5 subspecificity. In contrast, six of $29(21 \%)$ normal healthy DR2,DQw1 positive individuals carried the DRw16(Dw21),DQw5 haplotype. These data together with earlier results on the distribution of the DR4,DQw7 haplotype in patients with RA support the hypothesis that DQB1 chain polymorphism may be important in determining susceptibility to severe $\mathbf{R A}$.
\end{abstract}

Rheumatoid arthritis (RA) is a relatively common disease with a worldwide distribution. In the last decade a number of studies have investigated the genetic basis for RA. One major focus of these studies has been the attempt to identify genes within the class II region of the major histocompatibility complex - that is, HLA - which confer susceptibility or resistance for the disease. A strong association between HLA-DR4 and RA has been observed in most populations studied, though several exceptions have now been reported. ${ }^{1}$ In contrast, the incidence of DR2 is decreased in patients with RA, suggesting that this specificity may confer some protection against the disease. ${ }^{2-4}$ Evidence for the genetic susceptibility to RA also comes from the analyses of multiple case families, where HLA haplotype sharing in affected sibling pairs shows linkage of the susceptibility/resistance gene(s) to the HLA region. ${ }^{1}$

In white populations DR4 is found in about $66 \%$ of the patients with RA as compared with its incidence in $26 \%$ of healthy individuals. ${ }^{1}$ Investigations of the subtypes of DR4 showed a significant increase of Dw4 and Dw14 in white patients, and of Dw15 in Japanese patients. ${ }^{56}$ On the other hand, DR4 was not associated with RA in the Israeli population. ${ }^{7}$ Recently, we examined the extended DR4 haplotypes in patients with $R A$ and reported a significant increase of the $\mathrm{DR} 4, \mathrm{DQw} 7$ haplotype in patients with severe to moderate disease. ${ }^{89}$ Similarly, a significantly higher incidence of DR4,DQw7 haplotype in seropositive juvenile RA, severe forms of RA, and Felty's syndrome has also been described. ${ }^{10-13}$ Based on these observations, hypotheses for the molecular basis for associations between HLA class II antigens and RA have recently been proposed. ${ }^{14-16}$

The incidence of DR2 is significantly decreased in patients with RA as compared with normal controls. ${ }^{2-4}$ A number of subtypes of DR2 have been defined by serology and more finely by allorecognition in the mixed lymphocyte culture reactions. ${ }^{17-19}$ The subtypes of DR2 include DR2 long-DRw15 (Dw2 and Dw12) and DR2 short-DRw16 (Dw21=AZH, FJO,WJR,MN2, and Dw22=DB9,LD5a). Of these, Dw2, Dw12, and Dw21 are in linkage disequilibrium with different subspecificities of DQw1, DQw1.2(DQw6), DQwl.12(DQw6), and DQwl.AZH(DQw5), respectively. On the other hand, Dw22 is associated with DQw3TP(DQw7). ${ }^{19}$ DR2,DQwl haplotypes have, therefore, three subtypic (Dw2, Dw12, and Dw21) specificities. The definition and identification of these DR,DQ subspecificities have only been possible by Southern blot and nucleotide sequence analyses of cDNA clones. ${ }^{20-23} \mathrm{It}$ is evident from these data that these subspecificities of DR2,DQwl haplotypes are different at the genetic level. The distribution of these subspecificities in RA may be important for understanding the genetic basis of susceptibility/ resistance to the disease.

The objectives of this study were, therefore, to investigate the subtypes of $\mathrm{DR} 2, \mathrm{DQw} 1$ haplotypes in patients with $\mathrm{RA}$ by restriction fragment length polymorphism.

\section{Patients and methods}

Patients with RA who attended the rheumatology clinics at St Joseph's Hospital and McMaster 
University Medical Centre in Hamilton, and the Wellesley Hospital in Toronto, Ontario were studied. All patients had moderate to severe classical seropositive RA. ${ }^{8924}$ These patients had earlier been treated with non-steroidal antiinflammatory drugs and all had incomplete response requiring further treatment with sodium aurothiomalate as a disease remitting drug. ${ }^{9}$ Eighteen DR2 positive white patients with RA, who met these criteria, were studied. Of 106 patients with RA in Hamilton, 15 were positive for DR2. The remaining three patients were from the Wellesley Hospital. All DR2 positive patients were also positive for DQwl.

Nine Epstein-Barr virus transformed B lymphoblastoid cell lines, Dw2 (AMAI, DO208915, EA, MGAR, WT8), Dw12 (E4181324), Dw21 (KAS671, DEM), and Dw22 (RML), obtained from the Tenth International Histocompatibility Workshop, were used for the identification of subspecificities of DR2 haplotypes. Twenty nine unrelated healthy DR2,DQwl positive randomly selected individuals served as the control group.

Genomic DNA was prepared from peripheral blood lymphocytes and from lymphoblastoid cell lines. ${ }^{25}$ Samples of genomic DNA $(7 \mu \mathrm{g})$ were separately digested with 20 units of different restriction endonucleases, Bam HI, Eco RI, Eco RV, or Pvu II, for 18 hours at $37^{\circ} \mathrm{C}$ with buffer conditions specified by the manufacturer (Bethesda Research Laboratories) and were electrophoresed on a $0.9 \%$ agarose gel in TAE buffer $(40 \mathrm{mmol} / \mathrm{l}$ TRIS-acetate, $1 \mathrm{mmol} / \mathrm{l}$

Figure 1: Southern blots of DNA from three DR2 positive cell lines, digested with restriction endonucleases and hybridised with the $D Q \beta$ CDNA probe. Lane $1=$ DEM (Dw21, DR4); lane 2=AMAI (Dw2); lane $3=E 4181324(D w 12)$ ${ }^{*}=D w 21 ;^{* *}=D w 2,{ }^{* * *}=$ Dw12.
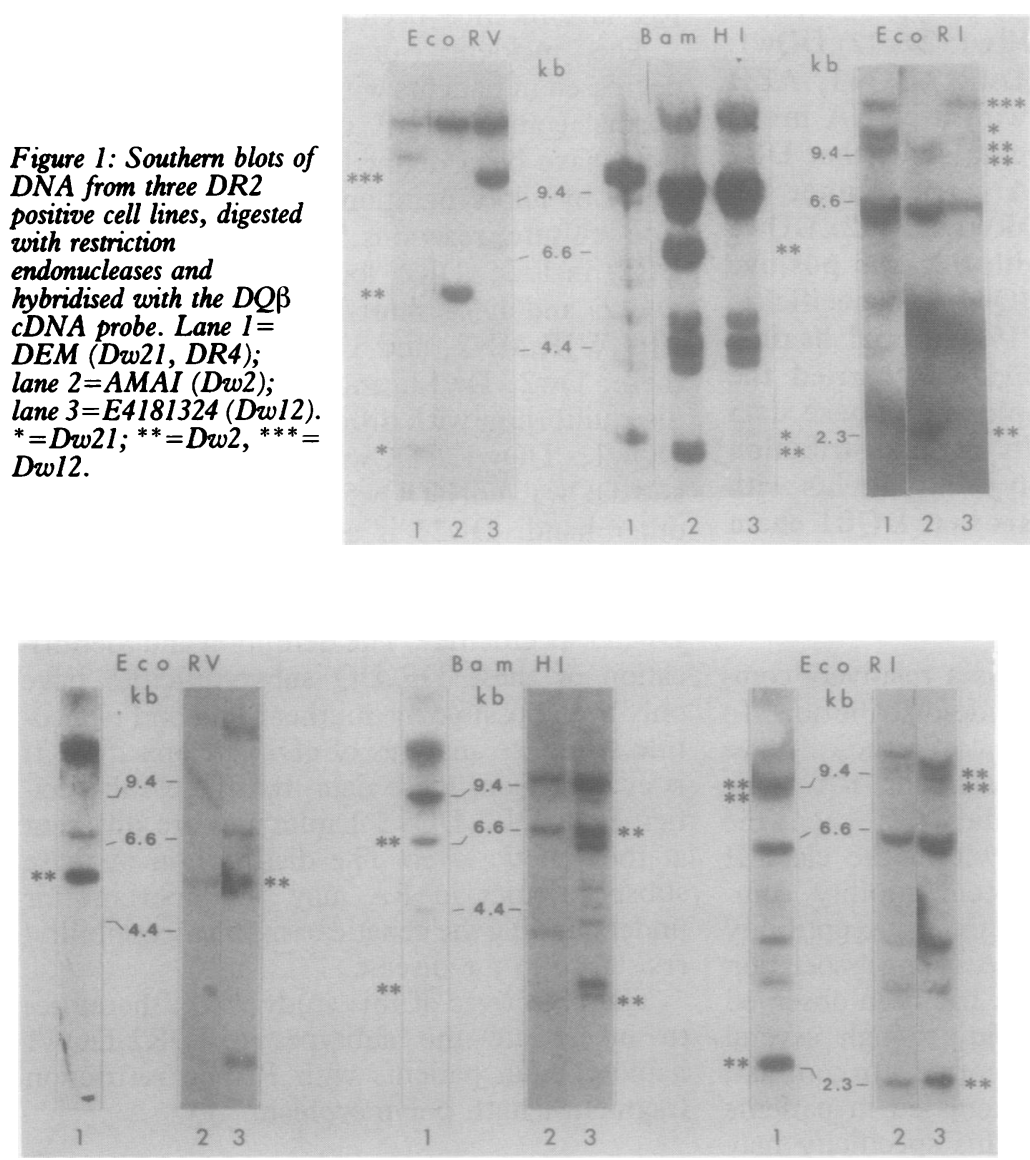

Figure 2: Southern blots of DNA from three DR2 positive patients with $R A$, digested with restriction endonucleases and hybridised with the $D Q \beta \mathrm{cDNA}$ probe. Lane $1=$ patient No 1 (Dw2); lane 2=No $2(D w 2, D R 3)$; lane $3=$ No $3(D w 2, D R I) .{ }^{* *}=D w 2$.
EDTA) for 18 hours at $50 \mathrm{~mA} .^{25}$ Molecular weight markers on each gel contained $1.5 \mu \mathrm{g}$ of Hind III digested lambda DNA (BRL). After electrophoresis, gels were denatured and neutralised before transfer (alkaline) to nylon membrane (Biotrace RP, Gelman) by Southern's method. ${ }^{26}$ After transfer, filters were prehybridised at $42^{\circ} \mathrm{C}$ and hybridised with oligonucleotide ${ }^{32} \mathrm{P}$ labelled $\mathrm{DQ} \beta \mathrm{cDNA}$ probe at $42^{\circ} \mathrm{C}$ for 48 hours. The DQ $\beta$ cDNA probe (pII- $\beta-1)$ (obtained from Dr P A Peterson, Uppsala ${ }^{27}$ ) was used as previously described by us. ${ }^{21}$ After hybridisation, blots were first washed for five minutes twice at room temperature with $2 \times$ SSPE $\left(0.3 \mathrm{~mol} / 1 \mathrm{NaCl}, 20 \mathrm{mmol} / 1 \quad \mathrm{NaH}_{2} \mathrm{PO}_{4} \cdot \mathrm{H}_{2} \mathrm{O}\right.$, $2 \mathrm{mmol} / \mathrm{l} \mathrm{EDTA}$ ); the filters were then washed for 15 minutes twice at $65^{\circ} \mathrm{C}$, once with $2 \times S S P E$ and $0.5 \%$ sodium dodecyl sulphate and once with $0.5 \times$ SSPE. The filters were autoradiographed with intensifying screens on Kodak XAR film at $-70^{\circ} \mathrm{C}$ for $3-10$ days.

Statistical analysis of the results was performed by the Fisher's exact test. ${ }^{28}$

\section{Results}

Hybridisation of DQ $\beta$ cDNA probe to Bam $\mathrm{HI}$, Eco RI, Eco RV, and Pvu II digested genomic DNA identified the three subspecificities (DRw15(Dw2),DQw6; DRw15(Dw12),DQw6; DRw16(Dw21),DQw5) of DR2,DQw1 haplotypes. Figure 1 shows the results from three $B$ cell lymphoblastoid cell lines, AMAI (Dw2), E4181324 (Dw12), and DEM (Dw21,DR4), with Eco RV, Bam HI, and Eco RI restriction endonucleases. Six bands, $5 \cdot 2 \mathrm{~kb}$ (Eco RV), $2 \cdot 8$ $\mathrm{kb}$ and $6.6 \mathrm{~kb}$ (Bam HI), and $2.4 \mathrm{~kb}, 9.2 \mathrm{~kb}$, and $9.6 \mathrm{~kb}$ (Eco RI), identify the DRw15(Dw2), DQw6 subtype; two bands, $11.0 \mathrm{~kb}$ (Eco RV) and $24.0 \mathrm{~kb}$ (Eco RI), identify the DRw15 (Dw12),DQw6 subspecificity, and three bands, $2.5 \mathrm{~kb}$ (Eco RV), $3.1 \mathrm{~kb}$ (Bam HI), and $16.5 \mathrm{~kb}$ (Eco RI), identify the DRw16(Dw21),DQw5 haplotype. Restriction fragment lengths identifying DRw16(Dw21), DQw5 subtypic specificity were also present in DR1 and some DRw6 haplotypes, suggesting cross reactivity between these specificities. ${ }^{20} 2223$ In addition, two Eco RI fragments $(9.6 \mathrm{~kb}, 24.0 \mathrm{~kb})$ were also present in DR4 positive cells, and one Bam HI band $(6.6 \mathrm{~kb})$ was also present in DR3 positive cells. These three subtypes of DR2 haplotypes were also identified after digesting genomic DNA with PVU II (data not shown). In addition, DQw7 associated DRw16(Dw22) differed from the DQw6 associated Dw2, Dw12, and DQw5 associated Dw21 subspecificities; this information is not provided in this study as all DR2 positive patients with $R A$ were positive for DQwl.

Genomic DNA from $18 \mathrm{DR} 2$ positive patients with RA was examined for the presence of these subtypic specificities. Figures 2 and 3 show the results for six of these patients. Five patients (lanes 1, 2, 3 (fig 2) and lanes 1, 2 (fig 3)) carried the DRw15 (Dw2),DQw6 subspecificity and one patient (lane 3 (fig 3)) was positive for the DRw15 (Dw12),DQw6 subspecificity. Of the remaining 12 DR2 positive patients with RA, 11 carried the DRw15(Dw2),DQw6 subspecificity and 
Figure 3: Southern blots of $D N A$ from three $D R 2$ positive $R A$ patients, digested with restriction endonucleases and hybridised with the $D Q \beta$ cDNA probe. Lane $1=$ patient No4 (Dw2, DRw6); lane $2=$ No $5(D w 2, D R 4)$; lane $3=$ No 6 (Dw12,DR3). ${ }^{* *}=D w 2,{ }^{* * *}=D w 12$.

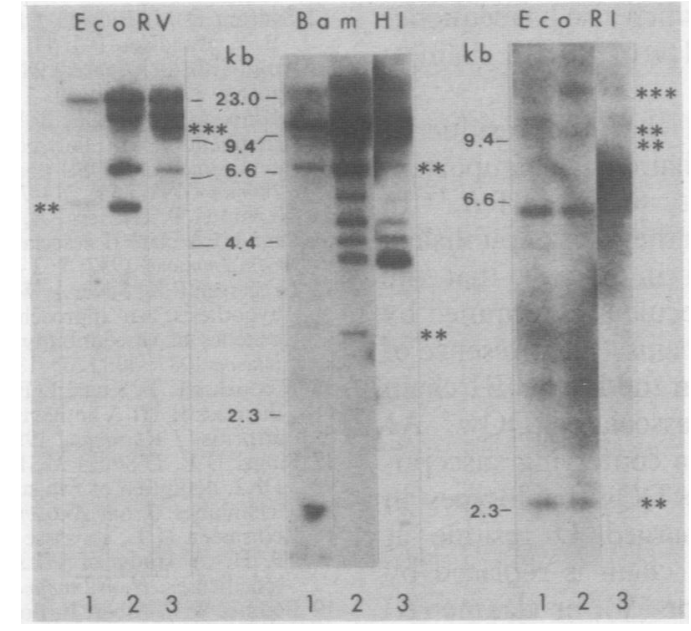

one was positive for the DRw15(Dw12).DQw6 specificity. None of the DR2 positive patients with RA carried the DRw16(Dw21),DQw5 subspecificity.

Of $29 \mathrm{DR} 2, \mathrm{DQw} 1$ positive unrelated controls, six typed as DRw16(Dw21),DQw5, two carried the DRw15(Dw12),DQw6 allele, and the remaining 21 typed as DRw15(Dw2),DQw6 (results not shown).

The incidence of the DRw16(Dw21),DQw5 subspecificity in DR2 positive patients with RA (0\%) was significantly (Fisher's exact test, p, two tailed $=0.04$ ) lower than in normal controls $(21 \%)$.

\section{Discussion}

The data in this study show that of 18 DR2 positive patients, 16 carried the DRw15(Dw2), DQw6 subtype and two patients were positive for the DRw15(Dw12),DQw6 subspecificity. None of these patients was positive for DRw16 (Dw21),DQw5. Of 29 unrelated healthy controls, 21 were positive for the DRw15(Dw2), DQw6 subtype, two typed as DRw15(Dw12), DQw6, and the remaining six carried the DRw16(Dw21),DQw5 subspecificity.

A diverse array of molecules contributes to the DR2 specificity, and this diversity is broadly distinguished by serology and by allorecognition in cellular reactions. ${ }^{17-19}$ The subspecificities of DR2 haplotypes have recently been defined by Southern blot analysis and nucleotide sequencing. ${ }^{20-23}$ In this investigation we used the restriction fragment length polymorphism approach to type for the subspecificities of DR2 haplotypes in patients with RA. The data show that all of the $18 \mathrm{DR} 2$ positive patients with RA were positive for the DRw15(Dw2,Dw12), DQw6 subtypes.

The subspecificities of DR2 are functionally important as polymorphism among $\mathrm{DQ} \beta$ alleles within DR2 results in subtype specific restriction. ${ }^{22}$ In addition, Jacobson et al used a panel of DR2 positive lymphoblastoid cell lines and showed that DR2(Dw2), OKT4+ cytotoxic.T lymphocyte clones specific for measles virus were restricted to Dw2 and Dw12 subspecificities of DR2. ${ }^{29}$ In view of the functional relevance of the subspecificities of DR2 several investigators have examined their incidence in a number of diseases, including insulin dependent diabetes, ${ }^{30} 31$ multiple sclerosis, ${ }^{32}$ and narcolepsy. ${ }^{33-35}$ In multiple sclerosis and narcolepsy all DR2 positive patients were positive for the Dw2 subspecificity. In contrast, most insulin dependent diabetic DR2 positive patients (nine of 11 in one study ${ }^{30}$ and six of nine patients in the other ${ }^{31}$ ) carried the Dw21 subtype. Wallin et al did not analyse their data for the distribution of subtypes of DR2 in patients with RA, ${ }^{36}$ but a subsequent examination of their data shows that all (8/8) DR2 positive patients (six unrelated, two members of two families) were positive for the Dw2 subtype. The results of our investigation extend these observations as all DR2 positive patients with RA carried the Dw2 or Dw12 subspecificity and none of these patients was positive for the Dw21 and Dw22 subtypes.

The incidence of DR2 in patients with $R A$ $(14 \%)$ in this study was lower than that in normal healthy controls (25\%). These results are thus in agreement with those published earlier by a number of investigators. ${ }^{1-4}$ The data in this study extend earlier results as the incidence of the DRw15,DQw6 subspecificity was similar $\left(\chi^{2}=0.71 ; p=N S\right)$ in normal controls (19\%) and in patients with RA (14\%).

In this investigation the DRw16(Dw21), DQw5 haplotype was present in six of 29 (21\%) unrelated DR2,DQw1 positive individuals. A similar incidence of this specificity in DR2 positive normal subjects has been found by others (in $2 / 11$ (18\%) French white subjects, ${ }^{30}$ in $7 / 40(18 \%)$ Israeli Jews, ${ }^{37}$ in $15 / 74(20 \%)$ Canadian white subjects ${ }^{38}$ ) and by us previously. ${ }^{17} 21$ As the number of DR2,DQw1 positive patients with RA in this study is small it is possible that the failure to find DRw16(Dw21), DQw5 positive patients might have been due to chance alone. In another study also, however, none of the eight DR2 positive patients with RA carried this subspecificity. ${ }^{36}$ In addition, the incidence of DRw16(Dw21),DQw5 allele in patients with RA in our investigation is significant (Fisher's exact test, $p$, two tailed $=0.04$ ) compared with its incidence in normal controls. It is possible therefore that the DRw16(Dw21) DQw5 subspecificity is not present in DR2 positive patients with RA.

Two groups of investigators proposed a shared epitope hypothesis implicating the involvement of DR4 B1 polypeptide in susceptibility to RA. ${ }^{14}$ 15 They suggested that the non-polar amino acid glutamine $(Q)$ at position 70 , in association with arginine $(R)$ or lysine $(K)$ at position 71, is predictive of disease susceptibility. The presence of these residues correlates with the association of Dw4, Dw14, and Dw15 with RA. A double substitution in the Dw10 allele results in two negatively charged amino acids, aspartic acid (D) and glutamic acid (E), in these positions and may thus explain the lack of association of this specificity with RA. This hypothesis, however, fails to explain the lack of increase in the incidence of DR4(Dw13) and of DR3 haplotypes in RA, both of which show sequence similarities to Dw4 and Dw14. In addition, a number of reports have failed to observe an increase in DRl in patients with 
$R A, 233940$ a specificity which also has sequence similarities to Dw4 and Dw14 DRB1 chain at positions 70 and 71 .

We recently proposed that the residue at position 57 in the $\mathrm{DQB} 1$ chain may be important in conferring susceptibility to severe RA. ${ }^{16}$ In addition, we suggested, on the basis of published data both in $\operatorname{man}^{41}$ and in mice, ${ }^{42}$ that the specificity of the DQ molecule is determined by both DQA1 and DQB1 chains. The presence of residue $\mathrm{D}$ at position 57 in the $\mathrm{DQw} 7 \mathrm{Bl}$ chain together with the DR4 associated DQw7 A1 chain may be important in conferring susceptibility to severe RA. The DQw haplotypes in which the negatively charged $D$ residue at position 57 in the DQB1 chain is replaced by non-polar serine $(S)$, valine $(\mathrm{V})$, or alanine $(A)$ may have a neutral or negative association with severe RA susceptibility. The results in this study on distribution of DR2 haplotypes in patients with RA support this hypothesis as (a) the DRw16(Dw21),DQw5 haplotype in which the DQB1 chain has residue $S$ at position 57 is absent in RA, and (b) the DRw15(Dw2,Dw12), DQw6 haplotype with residue $D$ in position 57 of the DQB1 chain is present in RA. As the DRw15,DQw6 associated DQAl chain is different from the DR4,DQw7 associated DQA1 chain it is likely that this specificity is not increased in patients with RA.

Rheumatoid arthritis is a heterogeneous disease. It is possible that both $\mathrm{DR}$ and $\mathrm{DQ}$ genes are important in conferring susceptibility to $R A-D R B 1$ in the mild form of the disease and $D Q B 1$ in severe $R A$, requiring sodium aurothiomalate as a disease remitting drug. We suggest that a multifactorial approach to the study of RA may serve to illuminate the associations between HLA antigens and the disease.

We are grateful to Dr D Gladman, The Wellesley Hospital, Toronto, for providing blood samples from three DR2 positive Toronto, for providing blood samples from three DR2 positive
patients with RA and to Dr J Singer, McMaster University, patients with RA and to Dr J Singer, McMaster University,
Hamilton, for analysis of the data. This work was supported by a Hamilton, for analysis of the data. This work was supported by a research grant from the A
Research Council of Canada.

1 Tiwari J L, Terasaki P I. HLA and disease associations. New York: Springer, 1985

2 Stastny P. Joint report, rheumatoid arthritis. In: Terasak P I, ed. Histocompatibility testing 1980. Los Angeles: UCLA P I, ed. Histocompatibility testing 1980. Los

3 Stastny P, Ball E J, Khan M A, Olsen N J, Pincus T, Gao X. HLA-DR4 and other genetic markers in rheumatoid arthritis. $\operatorname{Br} \mathcal{F}$ Rheumatol 1988; 27 (suppl 2): 132-8.

4 Jaraquemada D, Ollier W, Awad J, et al. HLA and rheumatoid arthritis: a combined analysis of $\mathbf{4 4 0}$ British patients. Ann Rheum Dis 1986; 45: 627-36.

5 Nepom G, Seyfried C, Holbeck S, Wilske K R, Nepom B S. Identification of HLA-Dw14 genes in DR4 ${ }^{+}$rheumatoid arthritis. Lancet 1987; ii: 1002-4.

6 Ohta N, Nishimura Y K, Tanimoto K, et al. Association between HLA and Japanese patients with rheumatoid between HLA and Japanese patients

7 Schiff B, Mizrachi Y, Orgad S, Yaron M, Gazit E. Association of HLA-Aw31 and HLA-DR1 with adult rheumatoid of HLA-Aw31 and HLA-DR1 with ad

8 Singal D P, D'Souza M Reid B, Bensen W G, Kassam Y B, Adachi J D. HLA-DQ beta-chain polymorphism in HLA-
D. DR4 haplotypes associated with rheumatoid arthritis. Lancet 1987; ii: 1118-20.

9 Singal D P, Bensen W G, Kassam Y B. HLA-DQ polymorphism in rheumatoid arthritis. Lancet 1988; i: 529.

10 Nepom B S, Palmer J, Kim S J, Hansen J A, Holbeck S L Nepom G'T. Specific genomic markers for the HLA-DQ subregion discriminate between DR4+ insulin-dependen diabetes mellitus and DR 4 + seropositive juvenile rheumatoid arthritis. F Exp Med 1986; 164: 345-50.

11 Grennan D M, Dyer P A. Immunogenetics and rheumatoid arthritis. Immunology Today 1988; 9: 33-4.
12 Sansom D M, Bidwell J L, Klouda P T, Amin S, Bradley B A, Maddison P J. HLA DQ polymorphism in rheumatoid arthritis. Lancet 1988; i: 58 .

13 Sansom D M, Bidwell J L, Maddison P J, Campion G, Klouda P T, Bradley B A. HLA DQ $\alpha$ and DQ $\beta$ restriction fragment length polymorphisms associated with Felty's fragment length polymorphisms associated with Felty's syndrome and DR4-positive

14 Nepom G T, Hansen J A, Nepom B S. The molecular basis for HLA class II associations with rheumatoid arthritis. $\mathcal{f}$ Clin Immunol 1987; 7: 1-7.

15 Gregersen P K, Silver J, Winchester R J. The shared epitope hypothesis. An approach to understanding the molecular genetics of susceptibility to rheumatoid arthritis. Arthritis Rheum 1987; 30: 1205-13.

16 McCusker C T, Singal D P. Molecular relationships between the class II HLA antigens and susceptibility to rheumatoid arthritis. $\mathcal{F}$ Rheumatol 1988; 15: 1050-3.

17 Singal D P, D'Souza M, Butler L. Heterogeneity of HLADR2: definition of two subtypes by serological and cellular techniques. Tissue Antigens 1985; 25: 283-9.

18 Reinsmoen N L, Layrisse Z, Betuel H, Freidel A C, Bach F H. A study of HLA-DR2 associated HLA-Dw/LD specificities. Hum Immunol 1984; 11: 105-15.

19 Bodmer $W$ F, Albert E, Bodmer J G, et al. Nomenclature for factors of the HLA system, 1987. In: Dupont B, ed. Immunobiology of HLA. New York: Springer, 1989: 72-9.

20 Segall $M$, Noreen $H$, Schluender L, Bach F H. DNA restriction fragment length polymorphism characteristics
for Dw subtypes of DR2. Hum Immunol 1986; 15: 336-43.

21 Singal D P, Butler L, Lamontagne L. Identification of subtypes of HLA-DR2 by Southern blot analysis. Tissue Antigens 1986; 28. 29-40.

22 Lee B S M, Bell J I, Rust N A, McDevitt H O. Structural and functional variability among DQ $\beta$ alleles of DR2 subtypes. Imunogenetics 1987; 26: 85-91.

23 Cohen D, Cohen O, Marcadet $\dot{A}$, et al. Class II HLA-DC $\beta$ chain DNA restriction fragments differentiate among HLADR2 individuals in insulin-dependent diabetes and multiple sclerosis. Proc Natl Acad Sci USA 1984; 81: 1774-8.

24 Ropes M W. Diagnostic criteria for rheumatoid arthritis: 1958 review. Ann Rheum Dis 1959; 18: 49-53.

25 Maniatis T, Fritsch E F, Sambrook J. Molecular cloning. New York: Cold Spring Harbor Laboratory, 1982.

26 Southern E M. Detection of specific sequences among DNA fragments separated by gel electrophoresis. F Mol Biol 1975; 98: 503-17.

27 Larhammer D, Schenning L, Gustafsson K, et al. Complete amino acid sequence of an HLA-DR antigen-like $\beta$-chain as predicted from the nucleotide sequence: Similarities with immunoglobulins and HLA-A, -B, -C antigens. Proc Nat Acad Sci USA 1982; 79: 3687-91.

28 Kleinbaum D G, Kupper L L, Morgenstern H. Epidemiologic research. Belmont, California: Wadsworth, 1982.

29 Jacobson S, Nepom G T, Richert J R, Biddison W E, McFarland $H$ F. Identification of a specific HLA-DR2 Ia molecule as a restriction element for measles virus-specific HLA class II restricted cytotoxic T cell clones. $\mathcal{F}$ Exp Med 1985; 161: 263-8.

30 Cohen N, Brautbar C, Font M, Dausset J, Cohen D. HLADR2-associated Dw subtypes correlate with RFLP clusters: Most DR2 IDDM patients belong to one of these clusters. Immunogenetics 1986; 23: 84-9.

31 Bach F H, Rich S S, Barbosa J, Segall M. Insulin-dependent diabetes-associated HLA-D region encoded determinants. Hum Immunol 1985; 12: 59-64.

32 Jacobson S, Sorrentino R, Nepom G T, McFarlin D E Strominger J L. DNA restriction fragment length polymorphism of HLA-DR2: correlation with HLA-DR2 associated functions. F Neuroimmunol 1986; 12: 195-203.

33 Marcadet A, Gebuhrer L, Betuel H, et al. DNA polymorphism related to HLA-DR2 Dw2 in patients with narcolepsy. related to HLA-DR2 Dw2 in pati

34 Honda Y, Juji T, Matsuki K, et al. HLA-DR2 and Dw2 in narcolepsy and in other disorders of excessive somnolence without cataplexy. Sleep 1986; 9: 133-42

35 Matsuki K, Maeda H, Juji T, et al. Taq I-generated HLA $\mathrm{DQ} \alpha$ polymorphism in Japanese patients with narcolepsy Imunogenetics 1988; 27: 87-90.

36 Wallin J, Carisson B, Storm H, Moller E. A DR4-associated DR-DQ haplotype is significantly associated with rheumatoid arthritis. Arthritis Rheum 1988; 31: 72-9.

37 Cohen N, Amar A, Oksenberg J R, Brautbar C. HLA-D clusters associated with DR2 and the definition of HLA-D "AZH": a new DR2 related HLA-D specificity in Israel. "Aissue Antigens 1984; 24: 1-9.

38 Mervart H, Mody M, Wong K H. HLA-DR2 subtypes. Tenth Intermational Histocompatibility Workshop Newsletter 1987; 2: 6.

39 Olsen N J, Callahan L F, Brooks R H, et al. Associations of HLA-DR4 with rheumatoid factor and radiographic severity in rheumatoid arthritis. Am $\mathcal{J}$ Med 1988; 84: 257-64.

40 Brautbar C, Naparstek Y, Yaron M, et al. Immunogenetics of rheumatoid arthritis in Israel. Tissue Antigens 1986; 28 8-14.

41 Rosenshine S, Cascino I, Zeevi A, et al. DQ $\alpha$ and DQ $\beta$ RFLP reveals the composition of the DQ molecule recognized by T-cell clones. Immunogenetics 1986; 23 $187-96$.

42 Lechler R I, Ronchese F, Braunstein N S, et al. I-A-restricted $T$ cell antigen recognition: analysis of the roles of $A \alpha$ and A 3 using DNA-mediated gene transfer. 7 Exp Med 1986; 163: 678-96. 